\title{
THE K-BAND HUBBLE DIAGRAM FOR X-RAY SELECTED BRIGHTEST CLUSTER GALAXIES
}

\author{
R.G. MANN ${ }^{1}$ AND C.A. COLLINS ${ }^{2}$ \\ ${ }^{1}$ Astrophysics Group, Imperial College \\ ${ }^{2}$ Astrophysics Group, Liverpool John Moores University
}

\section{Introduction}

The Hubble (magnitude-redshift) diagram for brightest cluster galaxies (BCGs) is a classic cosmological tool, widely studied because of the remarkably small dispersion ( $0.3 \mathrm{mag}$ ) in the absolute optical magnitudes of low redshift BCGs (Postman and Lauer 1995). Extending the BCG Hubble diagram to higher redshifts would greatly enhance its role as a cosmological probe, but this has been frustrated by several technical problems:

- the conventional means of cluster selection in the optical become increasingly compromised by projection effects at $z>0.1$

- at higher redshifts the interpretation of optical magnitudes becomes increasingly complicated by the effects of possible star formation.

We have overcomed both of these problems in a recent study (Collins and Mann in preparation):

- X-ray cluster selection minimises projection effects, yielding a BCG sample homogeneously selected out to $z \sim 1$

- by using the K-band we are primarily sampling the mature stellar population of the BCGs over that full range, and are insensitive to any possible bursts of star formation.

\section{Cluster Sample}

Our sample comprises BCGs in $48 \mathrm{X}$-ray selected clusters, principally drawn from the Einstein Extended Medium Sensitivity Survey (EMSS; Gioia and Luppino 1994), and supplemented by two ROSAT (Voges et al. 1996) clusters. The sample covers the redshift range $0.05<z<0.82$, and K-band 
images for them were obtained with the $3.8 \mathrm{~m}$ United Kingdom Infrared Telescope (UKIRT).

\section{Data Analysis}

We measured $\mathrm{K}$-band magnitudes for the sample within a constant metric aperture of diameter $50 \mathrm{kpc}\left(H_{0}=50 \mathrm{~km} \mathrm{~s}^{-1} \mathrm{Mpc}^{-1}, q_{0}=0.5\right)$, which were converted to absolute magnitudes using the $\mathrm{k}$-correction model of Glazebrook et al. (1995). This yielded an rms dispersion in the absolute magnitudes of 0.50 mags, greatly exceeding that found in previous optical (Postman and Lauer 1995) and K-band (Aragon-Salamanca et al. 1993) studies. Much of the scatter in absolute magnitudes comes from the lower- $z$ half of the sample. The rms dispersion for the higher- $z$ half $(z>0.225)$ is 0.28 mags, and that reduction is highly significant: Monte Carlo tests show that only $0.4 \%$ of randomly-selected 24 galaxy subsamples of our data set produce an rms dispersion lower than that. The physical cause of this effect is readily understood. The EMSS clusters were selected above an X-ray flux limit, so the lower redshift clusters tend to have lower X-ray luminosities, and we find a strong correlation between the K-band luminosities of our BCGs and the X-ray luminosities, $L_{\mathrm{X}}$, of their host clusters. Our results also confirm the existence of the $L_{\mathrm{m}}-\alpha$ relationship for BCGs, [where $L_{\mathrm{m}}$ is the metric luminosity within an aperture of size $r_{\mathrm{m}}$ and $\alpha$ is the logarithmic slope of $L_{\mathrm{m}}(r)$ at $r=r_{\mathrm{m}}$ ], as found by previous optical studies (Postman and Lauer 1995). If we correct our K-band magnitudes for their correlations with $L_{\mathrm{X}}$ and $\alpha$, then the rms dispersion of the 48 galaxy sample falls to 0.33 mags.

Furthermore, we find that the low- $L_{\mathrm{X}}$, low- $\alpha$ clusters at low redshift also tend to have lower numbers of neighbouring galaxies within a projected separation of $100 \mathrm{kpc}$. We infer, therefore, that, by selecting clusters above an $\mathrm{X}$-ray flux limit, we have included in our sample a low redshift $(z<0.3)$ population of first-ranked ellipticals in groups or poorer clusters which are different in kind from the $\mathrm{cD}$ galaxies in rich clusters conventionally envisaged as producing the classic BCG Hubble diagram. This is dramatically illustrated by the results of making an $L_{\mathrm{X}}$ cut to our sample: the 27 BCGs in our sample whose host clusters have $L_{\mathrm{X}}>2.3 \times 10^{44} \mathrm{erg} \mathrm{s}^{-1}$ in the $0.3-$ $3.5 \mathrm{keV}$ band yield an rms dispersion in absolute magnitudes of only 0.23 mags after correction for $L_{\mathrm{X}}$ and $\alpha$, and there is no evidence for a correlation between BCG luminosity and redshift in such a sample. Upon removal of one obvious outlier, which is a full magnitude brighter than the mean of this subsample and whose image comprises at least four very close components, one of which may well be a star, the rms dispersion falls to 0.17 mags, lower than any previously reported figure for BCGs. We are 
investigating the significance of this result for the study of BCGs and their host clusters, and for their use as cosmological probes.

\section{References}

Aragon-Salamanca A., et al., 1993, Mon.Not.R.astron.Soc., 262, 764

Collins C.A., Mann R.G., in preparation

Gioia I.M., Luppino G.A., 1994, Astrophys.J.Suppl., 94, 583

Glazebrook K., et al., 1995, Mon.Not.R.astron.Soc., 275, 169

Postman M., Lauer T.R., 1995, Astrophys.J., 440, 28

Voges W., et al., 1996, Astron.Astrophys., in press 